\title{
COMPORTAMENTO DE SEIS VARIEDADES DE POMELO (Citrus paradisi Macfad.) CULTIVADAS SOB IRRIGAÇÃO NA REGIÃO SEMI-ÁRIDA DE PERNAMBUCO ${ }^{1}$
}

\author{
ILDO ELIEZER LEDERMAN², JOÃO EMMANOEL FERNANDES BEZERRA³ ${ }^{3}$ MARTA ASSUNÇÃO ALVES ${ }^{4}$, \\ YGOR DA SILVA COELHO ${ }^{5}$, ALMIR PINTO DA CUNHA SOBRINHO
}

\begin{abstract}
RESUMO - O uso da irrigação nas regiões semi-áridas do Nordeste brasileiro tem viabilizado o cultivo comercial de inúmeras fruteiras, tais como a uva, a manga, a goiaba, o coco e a banana. Outras espécies, como o pomelo, ou grapefruit, vêm sendo ainda objeto de estudos e avaliações. Uma coleção formada de 06 variedades de pomelos (Citrus paradisi), de polpas com coloração rosa-avermelhada ('Marsh Foster', 'Star Ruby', 'Rio Red' e 'Red Blush') e amarelada ('Triumph' e 'Marsh Foster Nucelar'), foi implantada em 1993 na Estação Experimental de Ibimirim, localizada no município de Ibimirim, no Vale do Rio Moxotó - região semi-árida de Pernambuco. Contando com cinco plantas por parcela, a coleção foi conduzida sob irrigação localizada e as avaliações realizadas durante três anos (2000 a 2002). Os resultados demostraram alta variabilidade na produção de frutos, tendo a variedade 'Marsh Foster Nucelar' alcançado a produção média de 135,0 kg/planta, enquanto a 'Star Ruby' atingiu apenas 31,0 kg/planta. O peso médio dos frutos variou de 285,5g ('Red Blush') até um máximo de 401,6g na variedade 'Triumph'. O volume de suco extraído, os sólidos solúveis totais (SST), a acidez total titulável (ATT) e a relação SSS/ATT também apresentaram mudanças entre as variedades estudadas.
\end{abstract}

Termos para indexação: grapefruit, citros, produção, qualidade do fruto.

\section{BEHAVIOR OF SIX GRAPEFRUIT VARIETIES GROWN UNDER IRRIGATION AT THE SEMI-ARID REGION OF PERNAMBUCO - BRAZIL}

\begin{abstract}
The use of irrigation at the semi-arid region of Northeast Brazil has made feasible several commercial fruit trees crops such as grapevines, mango, guava, coconut, banana and others, like grapefruit, which actually is being studied. A collection formed by six grapefruit varieties showing a pink-red pulp color (Marsh Foster, Star Ruby, Rio Red and Red Blush) and a creamy color (Triumph and Marsh Foster Nucelar) and consisting of five plants/plot was installed in 1993 at the Ibimirim Experimental Station, located in the Moxotó river valley at the semi-arid region of Pernambuco - Northeast Brazil, under irrigation. Evaluations carried out during three years (from 2000 to 2002) have shown high yield variability among the varieties; with 'Star Ruby' reaching an average of $31.0 \mathrm{~kg} /$ tree and 'Nucelar Marsh Foster' yielding as much as 135,0 kg/tree. Mean fruit weight also varied, from $285.5 \mathrm{~g}$ (Red Blush) to $401.6 \mathrm{~g}$ in 'Triumph' variety. Fruit composition and quality measured through the total soluble solids (TSS), acidity (TA) and SST/TA ratio contents have shown high variations among grapefruit varieties studied.
\end{abstract}

Index Terms: Citrus paradisi, production, fruit quality.

\section{INTRODUÇÃO}

O uso da irrigação nas regiões semi-áridas do Nordeste brasileiro, associado à incorporação de novas tecnologias agrícolas e a um manejo cultural adequado, tem-se constituído num fator determinante para a viabilização de diversos cultivos frutícolas, dentre as quais, uva, manga, goiaba, coco, acerola, pinha, atemóia e graviola. Nessa região, a abundância de recursos hídricos, a predominância de um clima quente e seco na maior parte do ano e a alta incidência de irradiação solar permitem a obtenção de elevadas produtividades e frutos de excelente qualidade (Souza Leão, 2002).

Além das fruteiras atualmente cultivadas comercialmente, outras, como o sapotizeiro, a tamareira, a caramboleira, a pitangueira e o pomeleiro, ainda estão em fase de avaliação e estudos, podendo constituir em alternativas para a diversificação da fruticultura irrigada na região. Um zoneamento citrícola para o Brasil, proposto por Salibe (1977), enfatiza as possibilidades do cultivo de pomelos nas regiões Norte e Nordeste do País, haja vista as condições climáticas prevalecentes e muito propícias ao cultivo desses cítricos.

Os pomelos, também conhecidos por grapefruit nos países de língua inglesa, são frutas cítricas cujo cultivo apresenta grande importância econômica em países como os Estados Unidos, China, Cuba, Israel, África do Sul, México e Argentina (Coelho, 2002; Morton, 1987). No Brasil, a sua exploração está restrita a pequenos pomares, sendo a produção estimada em apenas 65 mil toneladas e destinada especialmente à exportação (Coelho, 2002). Apesar de os pomelos serem frutas bastante saudáveis, o seu teor de acidez é elevado para o paladar e hábitos brasileiros que, juntamente com um sabor doce e amargo, imprime característica bastante peculiar ao suco.

Dentre as numerosas variedades de pomelos, a 'Marsh Seedless' é a mais cultivada, apresentando entre suas principais características frutos com casca e polpa amareladas e ausência de sementes (Sinclair, 1972). Nos últimos anos vêm ganhando mercado, também, os pomelos pigmentados, de polpa rosada ou avermelhada, como a 'Foster', 'Redblush' e 'Star Ruby (Morton 1987).

No Brasil, os pomelos têm sido mais cultivados no Estado de São Paulo, que responde, praticamente, pela totalidade da produção comercial brasileira (Stuchi et al., 2003). Considerando, no entanto, que a região do vale do Rio Moxotó, em Ibimirim - Pernambuco, assim como boa parte do trópico semi-árido, reúne condições climáticas favoráveis ao cultivo desses cítricos, foram avaliados, neste trabalho, o comportamento produtivo e a qualidade dos frutos das variedades tradicionais, como também os pomelos pigmentados, de exploração comercial mais recente.

\section{MATERIALE MÉTODOS}

As borbulhas de 06 variedades de pomelos - quatro delas de polpa rosa-avermelhada ('Marsh Foster', 'Star Ruby', 'Rio Red' e 'Red Blush') e outras duas de coloração amarelada ('Triumph' e 'Marsh Foster Nucelar') - foram obtidas da Coleção de Citros da Embrapa Mandioca e Fruticultura, em Cruz das Almas - BA, e enxertadas sob o porta-enxerto de limão-cravo. As mudas enxertadas foram implantadas em 1993, na Estação Experimental de Ibimirim, da Empresa Pernambucana de Pesquisa Agropecuária - IPA, localizada no município de Ibimirim, região do Vale do Rio Moxotó. Localizada a $8^{0} 43^{\prime}$ de latitude sul, $37^{0} 32^{\prime}$ de longitude

\footnotetext{
1 (Trabalho 116/2004). Recebido: 07/10/2004. Aceito para publicações: 09/06/2005.

${ }^{2}$ Eng. Agr., PhD, bolsista do CNPq, Embrapa/IPA, Caixa Postal 1022, CEP:50761-000, Recife, PE. E-mail: ildo@ipa.br

${ }^{3}$ Eng.Agr., M.Sc., bolsista do CNPq, Empresa Pernambucana de Pesquisa Agropecuária - IPA. E-mail: emmanoel@ipa.br

${ }^{4}$ Eng.Agr.,M.Sc., Empresa Pernambucana de Pesquisa Agropecuária - IPA.

${ }^{5}$ Eng.Agr. Embrapa Mandioca e Fruticultura, Cruz das Almas - BA. E-mail: ygor@cnpmf.embrapa.br

${ }^{5}$ Eng. Agr. Embrapa Mandioca e Fruticultura, Cruz das Almas - BA
} 
oeste e altitude de $431 \mathrm{~m}$, a região é tipicamente semi-árida, apresenta temperatura média anual de $25^{\circ} \mathrm{C}$ e pluviosidade de $420 \mathrm{~mm} /$ ano, com clima BSWH, segundo Köppen. O solo é caracterizado como uma associação complexa de 'aluviões eutróficos' e 'solonetz solodizado', de textura média.

O plantio das mudas foi feito no espaçamento de $7,0 \mathrm{~m} \times 7,0 \mathrm{~m}$, com coleção de pomelos formada de 30 exemplares, sendo cada parcela constituída de cinco plantas. Anualmente, foram feitas adubações químicas com N-P-K, de acordo com a análise de solo, assim como capinas, pulverizações e podas de limpeza requeridas por essa cultura. Durante a estação seca da região - entre os meses de março á dezembro, e a intervalos de 3-4 dias, o sistema de irrigação por microaspersão foi ajustado de maneira a fornecer um volume d'água correspondente a 80 litros/planta.

$\mathrm{Na}$ avaliação da produção, foram computados os dados relativos ao período compreendido entre 2000 a 2002, sendo a colheita dos frutos maduros realizada em todas as plantas da parcela e os resultados expressos tanto em número de frutos como em produção ( $\mathrm{kg} /$ planta). O peso médio do fruto foi obtido dividindo-se a produção $(\mathrm{kg} /$ planta) pelo número de frutos colhidos.

A determinação dos principais componentes do fruto - casca polpa e semente - assim como as análises físicas e químicas foram feitas em uma amostra composta de 10 frutos, colhidos aleatoriamente na parcela. No Laboratório de Pós-Colheita de Frutas do IPA, procederam-se as seguintes determinações: 1) Composição centesimal - Inicialmente, os frutos inteiros foram pesados individualmente e, em seguida, utilizando-se de uma centrífuga, os seus principais componentes foram separados, e as frações obtidas, i.e., casca, polpa e semente, pesadas separadamente em uma balança analítica, e o resultado expresso em porcentagem. O rendimento do suco, extraído da polpa, foi mensurado em uma bureta e o resultado expresso em mililitros. 2) Análises químicas - o conteúdo de sólidos solúveis totais (SST), expresso em ${ }^{0}$ Brix, foi determinado por leitura direta em um refratômetro manual. A acidez total titulável (ATT) foi obtida por titulometria com $\mathrm{NaOH}$ a $0,1 \mathrm{~N}$ e o resultado expresso em porcentagem de ácido cítrico. Com base nestas informações, foi calculada a relação Brix / acidez.

\section{RESULTADOS E DISCUSSÃO}

As avaliações realizadas durante o período de 2000 a 2002 evidenciaram diferenças estatisticamente significativas quanto à produção de frutos entre algumas das variedades testadas. Ficou evidente, no entanto, a existência de uma alta variabilidade entre esses materiais (Tabelas 1 e 2). Enquanto a 'Star Ruby' alcançou a produção média de, apenas, 30,9 kg/planta, a 'Marsh Foster Nucelar' atingiu significativos $134,9 \mathrm{~kg} /$ planta.

TABELA 1 - Produção média (em número de frutos/planta) de seis variedades de pomelos (Citrus paradisi) cultivados sob irrigação em Ibimirim, região semi-árida de Pernambuco.

\begin{tabular}{c|ccccc}
\hline Varie dade & 2000 & $\mathbf{2 0 0 1}$ & $\mathbf{2 0 0 2}$ & Total & Média* $^{*}$ \\
\hline M. Foster & 18 & 290 & 258 & 566 & $188,66 \mathrm{bc}$ \\
Star Ruby & 39 & 127 & 191 & 357 & $119,00 \mathrm{c}$ \\
Rio Red & 97 & 247 & 272 & 616 & $205,33 \mathrm{bc}$ \\
Red Blush & 81 & 466 & 568 & 1115 & $371,66 \mathrm{ab}$ \\
Triumph & 42 & 276 & 469 & 787 & $262,33 \mathrm{bc}$ \\
Marsh F. Nucelar & 67 & 602 & 828 & 1497 & $499,00^{\mathrm{a}}$ \\
\hline
\end{tabular}

* Médias seguidas por uma letra não são significativamente diferentes, pelo Teste de Duncan.

Embora as produções obtidas nos anos em estudo estejam abaixo da expectativa, o potencial produtivo sugere a possibilidade de sucesso na exploração de pomelos, sobretudo em função da qualidade dos frutos. Por se tratar de pomeleiros com aproximadamente 10 anos de idade, admite-se que as plantas ainda não tenham atingido a estabilidade produtiva e, a permanecer o aumento gradativo verificado nesses três anos de avaliações, é possível que haja, nas próximas colheitas, um incremento significativo na produção.

O peso médio dos frutos, na maioria das cultivares estudadas, variou num pequeno intervalo de $285,5 \mathrm{~g}$ a $303,4 \mathrm{~g}$, todas estas apresentando diferença estatística em relação à 'Triumph', que alcançou 401,6g (Tabela 3). Os valores obtidos estão de acordo com os padrões estabelecidos no mercado internacional e demonstram que, sob as condições climáticas do semi-árido pernambucano, essas variedades expressam todo o seu potencial genético e reproduzem as suas principais características. Análises realizadas por Coelho et al. (1982) em frutos de 'Marsh Seedless, procedentes de quatro regiões brasileiras (Norte, Nordeste, Sul e Sudeste), demonstraram que os maiores frutos se originaram da região semi-árida de Pernambuco.

TABELA 2 - Produção (kg/planta) de seis variedades de pomelos (Citrus paradisi) cultivados sob irrigação em Ibimirim, região semi-árida de Pernambuco.

\begin{tabular}{c|ccccc}
\hline Variedade & $\mathbf{2 0 0 0}$ & $\mathbf{2 0 0 1}$ & $\mathbf{2 0 0 2}$ & Total & Média* \\
\hline M. Foster & 5,2 & 79,5 & 90,6 & 175,3 & $58,43 \mathrm{~b}$ \\
Star Ruby & 12,5 & 38,5 & 41,8 & 92,8 & $30,93 \mathrm{~b}$ \\
Rio Red & 34,2 & 61,6 & 93,5 & 189,3 & $63,1 \mathrm{ab}$ \\
Red Blush & 29,1 & 98,4 & 174,0 & 301,5 & $100,5 \mathrm{ab}$ \\
Triumph & 20,0 & 90.5 & 187,9 & 298,4 & $99,47 \mathrm{ab}$ \\
Marsh F. Nucelar & 23,6 & 133,4 & 247,6 & 404,6 & $134,87 \mathrm{a}$ \\
\hline
\end{tabular}

* Médias seguidas por uma mesma letra não são significativamente diferentes, pelo Teste de Duncan.

TABELA 3 - Peso médio dos frutos de seis variedades de pomelos (Citrus paradisi) cultivados sob irrigação em Ibimirim, região semi-árida de Pernambuco.

\begin{tabular}{c|ccccc}
\hline Variedade & $\mathbf{2 0 0 0}$ & $\mathbf{2 0 0 1}$ & $\mathbf{2 0 0 2}$ & Total & Média* \\
\hline M.Foster & 285,1 & 274,1 & 351,0 & 910,2 & $303,4 \mathrm{~b}$ \\
Star Ruby & 322,7 & 303,1 & 231,0 & 856,8 & $285,5 \mathrm{~b}$ \\
Rio Red & 297,3 & 249,4 & 343,9 & 890,6 & $296,87 \mathrm{~b}$ \\
Red Blusa & 358,0 & 211,1 & 306,4 & 875,5 & $291,83 \mathrm{~b}$ \\
Triumph & 476,2 & 327,9 & 400,7 & 1204,8 & $401,6 \mathrm{a}$ \\
Marsh .F. Nucelar & 350,4 & 221,6 & 299,0 & 871,0 & $290,33 \mathrm{~b}$ \\
\hline
\end{tabular}

* Médias seguidas por uma mesma letra não são significativamente diferentes, pelo Teste de Duncan.

Composição e qualidade do fruto - Os dados relativos à qualidade dos frutos revelaram maiores conteúdos de polpa para as variedades 'Star Ruby' e 'Marsh Foster Nucelar', com percentuais de 61,0 e 57,6\%, respectivamente (Tabela 4). Em alguns casos, todavia, esse alto percentual de polpa não se refletiu no conteúdo de suco, como, por exemplo, na variedade 'Star Ruby', que, embora tenha apresentado alto conteúdo de polpa, proporcionou o menor rendimento de suco $-28,3 \%$. Por outro lado, os maiores rendimentos de suco foram extraídos dos frutos das variedades Marsh Foster Nucelar e Rio Red, que atingiram, em média, os expressivos percentuais de 46,0 e 41,7\%, respectivamente. Coelho et al. (1982), em análises realizadas no pomelo Marsh Seedless cultivados em regiões com condições climáticas distintas (PE, BA, SP e RS), constataram menor rendimento em suco nos frutos procedentes do clima mais frio do Rio Grande do Sul.

No tocante à quantidade de sementes por fruto, observou-se que, à exceção do 'Marsh Foster', as demais variedades mostraram ausência de sementes ou, apenas, um número reduzido.

O maior conteúdo de sólidos solúveis totais foi observado no Star Ruby, cujo valor de $14,3^{\circ}$ Brix superou estatisticamente as demais variedades, enquanto os menores conteúdos foram registrados nas variedades Marsh Foster Nucelar e Rio Red, respectivamente (Tabela 4). Análises realizadas por Coelho (2002), em pomelos 'Marsh Seedless' importados e comercializados no mercado de Salvador (BA), apresentaram um conteúdo de SST de $8,9^{\circ}$ Brix, enquanto aqueles produzidos em diferentes regiões brasileiras variaram entre $8,3{ }^{\circ} \mathrm{Brix}$ 
(BA) e 10,4 ${ }^{\circ}$ Brix (RS) (Coelho et al.,1982).

Por outro lado, a variedade Star Ruby, além de apresentar maior conteúdo de SST, apresentou, também, maior acidez (2,7\% de ácido cítrico), resultando na mais baixa relação SST/ATT $(5,26)$. As demais variedades apresentaram teor de acidez que variou entre 1,58\% e 1,92\% (Tabela 4). Esses valores estão dentro da amplitude encontrada por Escalona et al. (1998) em quatro variedades de pomelos, na Venezuela, e nos frutos da variedade Marsh Seedless analisados por Coelho et al. (1982).

A relação Brix/acidez mostrou diferenças entre as variedades estudadas, sendo que a Marsh Foster, Triumph e Marsh Foster Nucelar - todas com relação Brix/acidez superiores a 6,0 - diferiram estatisticamente da Star Ruby. Esses valores estão próximos daqueles que foram divulgados por Escalona et al. (1998), na Venezuela, para quatro variedades de pomelos e, por Coelho (2002), em frutos importados de Marsh Seedless, que são comercializados no Brasil.

O presente estudo revela, com base nas avaliações do comportamento produtivo das seis variedades de pomelos e dos atributos de qualidade dos frutos, um destaque maior para as variedades Red Blush, de polpa pigmentada, e Marsh Foster Nucelar, de polpa amarelada. Ambas, além de alcançarem altas produtividades, proporcionaram, sob as condições climáticas do Vale do Rio Moxotó, produção de frutos de boa qualidade, expressa nos teores adequados de sólidos solúveis totais, acidez e relação Brix/acidez.

TABELA 4 - Composição e qualidade do fruto de seis variedades de pomelos cultivados em Ibimirim - Pernambuco $\left(^{*}\right)$

\begin{tabular}{|c|c|c|c|c|c|c|c|c|}
\hline \multirow[b]{2}{*}{ Variedade } & \multicolumn{3}{|c|}{ Composição centesimal (\%) } & \multirow[t]{2}{*}{$\%$ de suco $(*)$} & \multirow[b]{2}{*}{${ }^{\mathrm{o}}$ Brix } & \multirow[b]{2}{*}{$(*)$} & \multirow[b]{2}{*}{$\operatorname{Acidez}(\%)(*)$} & \multirow[b]{2}{*}{ Relação ${ }^{0}$ Brix/Acidez } \\
\hline & Casca & Polpa & Semente & & & & & \\
\hline Marsh Foster & 53,7 & 44,0 & 2,3 & $35,2 \mathrm{c}$ & $11,0 \mathrm{~b}$ & & $1,75 \mathrm{bc}$ & $6,28 \mathrm{a}$ \\
\hline Star Ruby & 38,8 & 61,0 & 0,2 & $28,3 \mathrm{~d}$ & $14,2 \mathrm{a}$ & & $2,70 \mathrm{a}$ & $5,26 \mathrm{~b}$ \\
\hline Rio Red & 48,6 & 51,4 & 0,0 & $41,7 \mathrm{ab}$ & $9,3 \mathrm{~d}$ & & $1,58 \mathrm{~cd}$ & $5,88 \mathrm{ab}$ \\
\hline Red Blush & 51,6 & 48,3 & 0,1 & $39,4 \mathrm{bc}$ & $10,9 \mathrm{~b}$ & & $1,92 \mathrm{~b}$ & $5,67 \mathrm{ab}$ \\
\hline Triumph & 51,8 & 47,5 & 0,7 & $38,5 \mathrm{bc}$ & $10,4 \mathrm{c}$ & $c$ & $1,68 \mathrm{~cd}$ & $6,19 \mathrm{a}$ \\
\hline Marsh .F. Nucelar & 42,3 & 57,6 & 0,1 & $46,0 \mathrm{a}$ & $9,22 \mathrm{~d}$ & $d$ & $1,51 \mathrm{~d}$ & $6,10 \mathrm{a}$ \\
\hline
\end{tabular}

(*) Médias seguidas por uma mesma letra não são significativamente diferentes, pelo Teste de "Duncan".

\section{CONCLUSÕES}

1) O cultivo de pomelos na região semi-árida do vale do Rio Moxotó, em Pernambuco, permite a obtenção de produtividades razoáveis e frutos de boa qualidade.

2) As variedades Red Blush, de polpa avermelhada, e a Marsh Foster Nucelar, de polpa amarelada, destacaram-se por reunirem melhores características produtivas e atributos de qualidade.

3) O peso médio dos frutos, para a maioria das variedades estudadas, variou num pequeno intervalo de $285,5 \mathrm{~g}$ a $303,4 \mathrm{~g}$.

4) Três das variedades testadas - Marsh Foster, Triumph e Marsh F. Nucelar - apresentaram uma relação Brix/acidez superior a 6,0.

\section{REFERÊNCIAS}

COELHO, Y.S.; POMPEU JR., J.; DORNELLES, C. M.M.; SOUZA, E. S.; NUNES, R.F M. Maturação e qualidade do pomelo 'Marsh Seedless' no Brasil. In: CONGRESS OF AMERICAN SOCIETY FOR HORTICULTURAL SCIENCE, 29., 1982, Campinas. Proceedings... p..163-170.
COELHO, Y. Frutas cítricas importadas no mercado de Salvador, Bahia. Bahia Agrícola, Salvador, v.5, n.2, p.29-33.2002.

ESCALONA, G.L.; MONTEVERDE, E.E.; RANGEL, L.; ESPINOZA, M. Evaluación de la calidad en frutos de naranjos, mandarinos, pomelos e híbridos injertados sobre 'Cleopatra'. Bioagro, Viçosa, v.10, n.2, p.35-39. 1998.

MORTON, J. Grapefruit-Citrus paradisi. In: MORTON, J.F. Fruits of warm climates. Miami: Agscience, 1987. p.152-158.

SALIBE, A. A . Clima e solo - zoneamento da citricultura brasileira. In: CURSO DE ESPECIALIZAÇÃO EM CITRICULTURAEM NÍVEL DE PÓS-GRADUADO. Recife: SUDENE/UFRPE, 1977.p.47-49.

SINCLAIR, W.B. The grapefruit: its composition, physiology, and products. Davis: University of California, 1972.660p.

SOUZA LEÃO, P C. de. Uva de mesa: produção - aspectos técnicos. Petrolina: Embrapa Semi-Árido; Brasília: Embrapa Informações Tecnológicas, 2001. 128p. (Frutas do Brasil, 13).

STUCHI, E.S.; DONADIO, L.C.; SEMPIONATO, O.R. Evaluation of 10 rootstocks for 'Marsh' seedless grapefruit in São Paulo, Brazil. In: INTERNATIONAL SOCIETY OF CITRICULTURE CONGRESS, 9., 2003. Orlando. Proceedings... v.1, p.586. 